\title{
A Framework for Establishing Early Warning System for Working in Hot Environments
}

\author{
W. Yi' ${ }^{\mathrm{a}}$, J. Zhu ${ }^{\mathrm{b}}$, X. Liu ${ }^{\mathrm{b}}$, X. Wang ${ }^{\mathrm{b}}$ and A.P.C. Chan \\ ${ }^{a}$ Department of Building and Real Estate, The Hong Kong Polytechnic University, Hong Kong, China \\ ${ }^{b}$ School of Built Environment, University of Curtin, Australia \\ E-mail: yiwen96@163.com,junxiang.zhu@postgrad.curtin.edu.au,xin.liu@curtin.edu.au, \\ xiangyu.wang@curtin.edu.au, albert.chan@polyu.edu.hk
}

\begin{abstract}
Heat stress is a well-known occupational hazard, and climate change together with the increased frequency and intensity of extreme heat events has made risks more severe and widespread. The liquefied natural gas (LNG) construction industry is found to be more susceptible to heat stress than other industries because LNG workers are subjected to heat stress not only from outdoor strenuous physical work but also in confined working spaces which could be even worse. Traditional management of heat stress are by and large some "dos and don'ts" and are not based on scientific measurements. The continuing high frequency of heat related incidents in the construction LNG industry calls for better approaches to detect impending attacks of heat stress. The purpose of study is to develop a smart work site against hot climates using modern technologies. The proposed system could be automated by information communication technology, sensor technology, Radio Frequency Identification (RFID) technology, to protect the wellbeing for those who have to work in hot environments. A Bayesian networks model could be established to evaluate the heat strain of workers. 3D environment map of a LNG site could be simulated by the readings of environmental sensors and trilinear interpolation. The work duration, location, and workplace heat exposure of workers could be monitored during the work and warning alerts would be sent to the workers when certain threshold exceeds. The proposed smart work site for early warning of heat stress would be of tremendous value in better safeguarding workers' health and safety by reducing the occurrences of heat stress on site.
\end{abstract}

Keywords -

Heat stress; Health and safety; Liquefied natural gas (LNG) construction industry; Early-warning system

\section{Introduction}

Liquefied natural gas (LNG) industry is one of the most rapid growing energy market trades. As a large, dynamic, and complex sector, it creates employment for millions of people worldwide. It is estimated that the LNG workforce in Queensland could increase to 17,000 workers in 2024 based on 59,000 wells and six LNG trains [1]. Construction projects of LNG plant involving assembling and installing of pipes, pumps, vessels, storage tanks, cooler and electronic system is complicated, which would cause a higher risk of workrelated illnesses and accidents. Considering the complicated nature and the large scale, health and safety of LNG workers needs to be paid greater attention.

Heat stress is recognized as major occupational hazard in many workers [2]. In a broader sense of comparison between the East and the West, an average of 13.8 workers per year died from heat related disorders in Japan between 1991 and 2001 [3]. A cohort study among 58,495 Thai workers indicated that approximately $20 \%$ of them experienced occupational heat stress that significantly related to injuries and illnesses [4]. In the United States, 423 workers in agricultural and non-agricultural industries were reported to have died from exposure to environmental heat between 1992 and 2006 [5]. Many occupational settings are commonly encountered in extreme hot environments, including outdoor cleaning and horticulture, iron manufacturing, mining, firefighting, military, and catering, and glass factories. Liquefied natural gas (LNG) workers are at high risk of heat stress not only from outdoor strenuous task, but also in confined working spaces, which could be even worse. More importantly, the four existing and six proposed LNG facilities in Australia are built in Zone 1 characterised as high humid summer proposed by the Building Code of Australia. Zone 1 represents a climate with hot humid summer and warm winter. As the 


\section{Conference topic}

number of oil and gas developments increase in a hot and humid environmental condition in Australia, heatrelated disorders and heat stress prevention are of critical importance for the Australian LNG industry.

To protect workers from the risk of heat stress, a number of guidelines and initiatives have been put in place to promote health and safety in the workplace including proper work-rest schedule, ventilation facilities at work or rest places, provision of portable water, personal cooling system [6]. However, these recommendations are largely based on regulations, which is considered as a reactive approach to manage heat stress. No systematic studies has been initiated to early warning and safety evaluation of heat stress recognition, assessment, control and management of individual behaviour. Advanced technologies and devices have been regarded as an effective and proactive method to enhance on-site safety performance. For example, $\mathrm{Wu}$ et al. argued that by using RFID sensor networks for tracking near-misses, onsite accidents can be avoided, hence improving safety performance [7]. Cheng et al. adopted physiological status monitoring (PSM) technology to monitor safe and unsafe behaviour of workers [8]. Therefore, the research aims to develop an early-warning system for heat stress using modern technologies and Bayesian network as the analytical algorithm.

\section{Framework for Early-warning System of Heat Stress}

The aim of the early-warning system is to reduce the occurrence of heat-related incidents on site. The research will combine the assessment of workers' heat strain, simulation of 3D site environment, and establishment of early-warning system. The framework of the early-warning system of heat stress in shown in Figure 1.

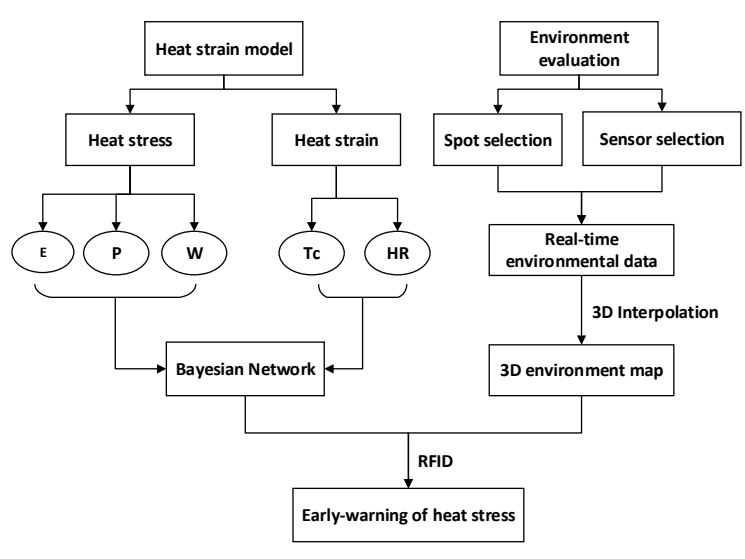

Figure 1 Framework of the early-warning for heat stress

Note: $\mathrm{E}$ is environmental data (i.e., air temperature, relative humidity, wind speed, solar radiation); $\mathrm{P}$ is personal information (i.e., age, percentage of body fat, smoking habit, alcohol drinking habit); $\mathrm{W}$ is workrelated data (i.e., work duration, work intensity); Tc is core temperature $\left({ }^{\circ} \mathrm{C}\right)$; HR is heart rate $(\mathrm{bpm})$.

\subsection{Stage 1: Heat Strain Model}

Heat stress is defined as "the net heat load to which a worker may be exposed from the combined contributions of metabolic cost of work, environmental factors and clothing requirements" [9]. The effects of working in hot weather can potentially have a severe influence on the welfare of workers if the risks have not been properly managed or considered. It may result in an increase in heart rate, body core temperature, and sweating. When subjected to a hot environment, heart rate will increase to move blood and dissipate heat from the heart, lungs, and other vital organs to the skin [10] and [11]. If the excessive heat is not fully dissipated from the body, the body will store heat and as a result core temperature will rise. The common heat-related disorders are heat fainting, heat exhaustion, and heat stroke. The heat strain of workers is assessed by their body core temperature and heart rate. Heat exhaustion is a moderate heat illness in which core temperature is between $38^{\circ} \mathrm{C}$ and $40^{\circ} \mathrm{C}$ [12]. Heat stroke is the most severe heat illness when core temperatures exceed $40^{\circ} \mathrm{C}$ [13]. Earlier studies reveal that human heart rate is in the range of 130 and $160 \mathrm{bpm}$ when he/she performs exercise in hot environments. The American Conference of Governmental Industrial Hygienists (ACGIH) has recommended the threshold limit values (TLVs) for heat stress and suggested the limit for heart rate is $180 \mathrm{bpm}$ minus the age of an individual lasting no more than three minutes [14]. Furthermore, physiological 
researches have demonstrated heat strain are associated with personal, work-related, and environmental factors [15].

Field studies will be conducted during summer time in different work sites. Outdoor workers without flu in the week prior to participation, without a history of diagnosed major health problem such as diabetes, hypertension, cardiovascular disease and regular medication intake will be invited to participate in the field study. Subjects will be asked to provide personal information on age, alcohol drinking habit, sleeping habit. Their height, weight, percentage of body fat, resting heart rate, blood pressure, and thermal insulation of their clothing will be measured. During the test, the workers will perform tasks as their usual daily routine. Wet bulb globe temperature, integrating all four elements of the thermal environment (i.e., air temperature, relative humidity, air velocity, and solar radiation), will be measured by sensors mounted on safety helmet at regular time intervals. Heart rate and energy consumption will be recorded every 10 s by a heart rate belt (Polar Vantage XL, Finland). Core temperature will be measured by an ingestible telemetric temperature system, which consists of a commercial temperature pill and a compact data receiver (CorTrack ${ }^{\mathrm{TM}}$, HQInc., Palmetto, Florida, USA). A sensor inside the temperature pill could transmit a continuous radio wave that varies with temperature. The data logger will receive and store this signal accordingly.

RFID integrated with mobile communication system will be adopted to track subjects' positions in outdoor environment. Subjects' positions will be sent back to a tracking server at defined time interval. Subjects' realtime positions will be displayed in a Geographic Information System (GIS) system. The position, physiological and environmental information collected by RFID, CorTrack ${ }^{\mathrm{TM}}$ and environmental sensors, respectively, will be stored in a geospatial database for further analysis together with site structure and development drawings, plans. The relationship between heat strain (core temperature and heart rate) and factors affecting heat strain (e.g., personal characteristics, environmental variable, work-related data, and work location) will be established by the Bayesian network model.

\subsection{Stage 2: Environment Evaluation}

Environmental conditions in various work locations are at different levels of heat stress within a work site, the aim of stage 2 is to simulate the environment condition of a LNG site. WBGT is calculated by the display of three thermometers on globe temperature, wet bulb temperature, and dry bulb temperature. Sampling locations for WBGT will be optimized by Kriging to better capture the spatial variation of the environmental condition [16]. To monitor the WBGT at different locations of a LNG site, several WBGT sensors packed by senor module (that involved globe temperature, wet bulb temperature, and dry bulb temperature sensors), Global System for Mobile Communications (GSM) module, and microcontroller will be deployed [17]. The microcontroller converts the signal from analog to digital form, issues the data to the GSM module, and orchestrates the routing of data through the network. Thus, real-time environmental data sampled from WBGT sensor can be transmitted to a server via GSM. Based on the timely updated WBGT data at different locations, the 3D environmental map of a LNG site will be generated through the trilinear interpolation.

\subsection{Stage 3: Early-warning System}

The purpose of the early-warning system is to safeguard the wellbeing of LNG workers while working in hot environment. The proposed early warning system is capable of collecting data on (a) instant WBGT at different zones of LNG sites; (b) location of workers; (c) work duration; and (d) personal information of workers. It could be automated by integrating smart sensor technology, RFID technology, and information communication technology. It is used to monitor the environmental condition of the whole LNG site, track the location of LNG workers, and alert workers the risks of heat stress by alarming sounds. Attendance of LNG workers are recorded automatically when workers walk through the entrance and enter the site with their helmets. Basic information (e.g., age, percentage of body fat, smoking habit, alcohol drinking habit, job nature, etc.) could be transmitted to the site office. The real-time location of workers in different zones of the LNG site could be monitored by the RFID. According to location of workers, the level of heat stress the workers exposed to can be obtained by the 3D environmental map. Based on these personal, workrelated, and environmental data, workers' heat strain can be evaluated according to the Bayesian network model.

\section{A Bayesian Network Approach to Assess Heat Stress}

\subsection{Concepts of a Bayesian Network}

A Bayesian Network is a network used to model the uncertainty relationships of the actual world with probabilities [18]. It has been applied to numerous studies involving human physiology, such as predicting 


\section{Conference topic}

individuals' physiological responses in an interactive virtual environment [19], estimating complex multitask operators' workload from their physiological and psychological features [20], and predicting personalised optimal comfort temperature for individuals [21]. Two parts are contained in a Bayesian Network: a body and the knowledge. The body of a Bayesian network is the structure made up of two components. One is nodes which represent variables of interest and the other is arcs pointing from one node to another that indicate causal relationships among nodes. Nodes with arcs orienting from them to other nodes are called parents nodes and those with arcs pointing to them are called children nodes. Also, a node is a root node when it has no parent nodes, and one node are called leaf node when it doesn't have any children nodes. A Bayesian Network's body formed by nodes and arcs is also referred to as a Directed Acyclic Graph (DAG), in which you can never get back to a previous node following the direction of arcs [22, 23].

The knowledge of a Bayesian Network is the posterior probability distribution of each node in the network. When the data supporting the Bayesian Network are discrete, i.e. the variables have several definite statuses, the posterior probability distribution comes in the form of a Conditional Probability Table (CPT), which records the probabilities of every possible combination of its parent nodes' statuses. In practice, if the data are continuous, they tend to undergo discretization before they can be used to build a Bayesian Network. This is because the continuous-data situation is far more complicated than a discrete one to compute and to understand. The equation for the joint probability distribution of all variables in a Bayesian Network is shown as follows (Neapolitan, 2004):

$$
P\left(X_{1}, X_{2}, \ldots, X_{n}\right)=\prod_{i}^{n} P\left(X_{i} \mid \text { Parents }\left(X_{i}\right)\right)
$$

Where $P\left(X_{1}, X_{2}, \ldots, X_{n}\right)$ refers to the joint probability distribution of node $X_{1}, X_{2}, \ldots, X_{n}$, Parents $\left(X_{i}\right)$ means all the parents nodes of node $X_{i}$. $P\left(X_{i} \mid\right.$ Parents $\left.\left(X_{i}\right)\right)$ stands for the conditional probability distribution of $X_{i}$, provided its parents [18, 24-26].

\subsection{Bayesian Network for Heat Strain}

A number of International Organization for Standardization (ISO) recommended indicators of heat strain as body core temperature and heart rate [27]. Evaluation of heat stress is generally determined through meteorological parameters that enable the estimation of the influence of several environmental factors on thermal comfort and physiological ability
[28]. WBGT, integrating the effects of temperature, humidity, air velocity, and solar radiation, is by far the most widely used index of heat stress. Apart from the meteorological parameter, which can be measured by a comprehensive environmental index, heat strain is also highly correlated with work-related factors. It includes the type of exercise habit and other personal factors such as age, physique, percentage of body fat, and hydration level (Chan et al. 2012). Previous studies also demonstrated that the effects of aging on body fat [29]. Therefore, a Bayesian Networks model including these information was built to assess heat strain of workers (Figure 2).

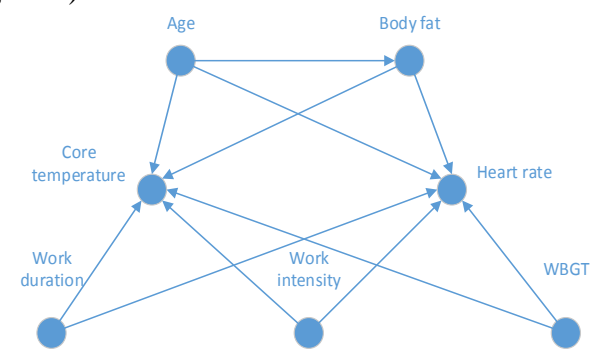

Figure 2 Bayesian Network in heat strain of workers

\section{3D Mapping of Work Environments}

In reality, environmental information can only be collected at particular spots. Those data are scattered point data. However, people work and live in a 3D space. As a useful approach to derive values for unknown points from a discrete set of known values, 3D-interpolation is employed to obtain spatially continuous and gridded data.

Many interpolation methods have been developed, including nearest neighbour, averaging, linear, and so on. However, for spatial data like temperature, Kriging, which is applied in Geosciences like Geology, Soil Science, Geography etc., has been mathematically proven to be the most stable linear unbiased estimation technique [30, 31], as traditional interpolation methods are mathematical only, while the Kriging is built on a model of stochastic spatial variation that fits well with reality [32] . The basic Kriging equation is shown as

$$
\hat{Z}(\mathrm{p})=\sum_{i=1}^{n} w_{i} Z_{i}\left(p_{i}\right)
$$

Where $\hat{Z}(\mathrm{p})$ is the unknown value that needs to be estimated at the position $p$ with known points $p_{i}$ and corresponding known values $Z_{i}\left(p_{i}\right)$ as its neighborhood. $n$ stands for the number of neighbouring known points used to krige the estimate, and $w_{i}$ is the weight for point $p_{i}$. 
Equations (3) and (4) make $\hat{Z}$ the best linear unbiased estimator [32, 33].

$$
\begin{gathered}
E(\hat{Z}-Z)=0 \\
E(\hat{Z}-Z)^{2} \text { is minimum }
\end{gathered}
$$

$\hat{Z}-Z$, the estimation error, indicates the similarity between $\hat{Z}$ and $Z, E(\hat{Z}-Z)^{2}$ is the mean square error and $E$ is the expectation.

Figure 3 shows an example of scattered temperature measurement on site. The points in the 3D space represent the positions of sampling collected by the smart sensors. Different colours stand for various degrees of temperature. Environment conditions at different locations are varying. Points in different colours denote different WBGT values. Through 3D interpolation, as shown in Figure 4, we transform those irregular points into spatially continuous WBGT data, which is much easier to perceive and understand.

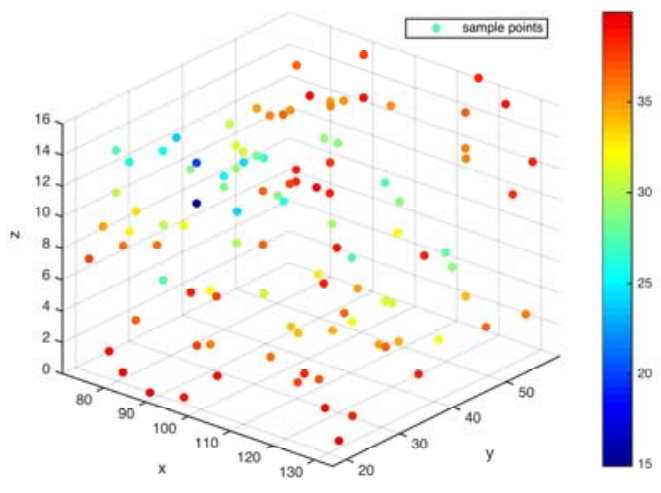

Figure 3 Sample of temperature measures in a 3D space

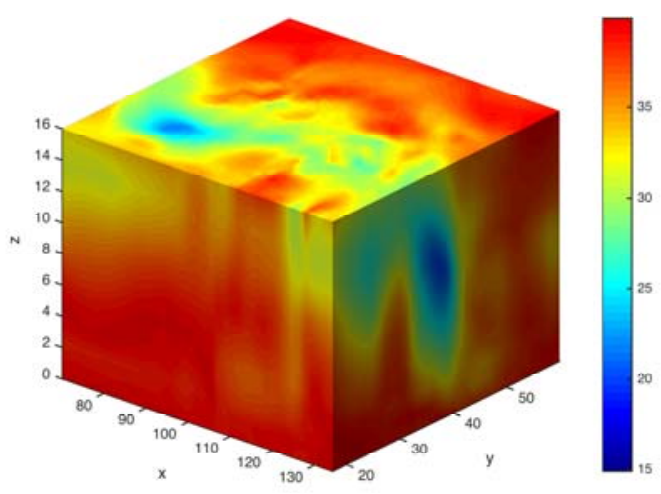

Figure 4 3D temperature field interpolated from scattered temperature samples

\section{Conclusion}

LNG workers are often exposed to severe environmental conditions, especially in North Australia, where most nature resource companies are located and the occupational settings are commonly in extreme hot environments. This increases the risk of heat stroke, injury and death. To protect workers from the risk of heat stress, a number of guidelines and initiatives have been put in place to promote health and safety in the workplace. However, most of these guidelines are largely depend on the specific regulations of workers' own behaviours. This study proposed an automatic way to monitor and early-warn any potential heat stress risk. The significant contributions of this study include: (1) a framework of interactive heat strain and environment evaluation model based on sensors technologies and mobile communication system; (2) a Bayesian Network in heat strain of workers; and (3) 3D interpolation model of environment condition on the work site.

\section{References}

[1] Queensland, E.S., Queensland CSG to LNG industry workforce plan: operations and maintenance 2014-2034. 2013.

[2] Chan, A.P. and Yi, W., Heat stress and its impacts on occupational health and performance. Indoor and Built Environment, 25(1): p. 3-5, 2016.

[3] Inaba, R. and Mirbod, S.M., Comparison of subjective symptoms and hot prevention measures in summer between traffic control workers and construction workers in Japan. Industrial health, 45(1): p. 91-99, 2007.

[4] Tawatsupa, B., et al., Association between heat stress and occupational injury among Thai workers: Findings of the Thai Cohort Study. Industrial health, 51(1): p. 34-46, 2013.

[5] Control, C.f.D. and Prevention, Heat-related deaths among crop workers--United States, 1992--2006. MMWR: Morbidity and mortality weekly report, 57(24): p. 649-653, 2008.

[6] Council, C.I., Guidelines on site safety measures for working in hot weather. Version 2, 2013.

[7] Wu, W., et al., Towards an autonomous real-time tracking system of near-miss accidents on construction sites. Automation in Construction, 19(2): p. 134-141, 2010.

[8] Cheng, T., et al., Data fusion of real-time location sensing and physiological status monitoring for ergonomics analysis of construction workers. 


\section{Conference topic}

Journal of Computing in Civil Engineering, 27(3): p. 320-335, 2012.

[9] Di Corleto, R., Firth, I., and Coles, G., Heat stress standard \& documentation developed for use in the Australian environment. Australian Institute of Occupational Hygienists, 2003.

[10] Brouha, L. and Maxfield, M.E., Practical evaluation of strain in muscular work and heat exposure by heart rate recovery curves*. Ergonomics, 5(1): p. 87-92, 1962.

[11] Wang, F., et al., Can the PHS model (ISO7933) predict reasonable thermophysiological responses while wearing protective clothing in hot environments? Physiological measurement, 32(2): p. 239, 2010.

[12] Howe, A.S. and Boden, B.P., Heat-related illness in athletes. The American Journal of Sports Medicine, 35(8): p. 1384-1395, 2007.

[13] McDermott, B.P., et al., Exertional heat stroke basics: What strength and conditioning coaches need to know. Strength \& Conditioning Journal, 30(3): p. 29-32, 2008.

[14] Worldwide, A., TLVs and BEIs-Threshold limit values for chemical substances and physical agents biological exposure indices. ACGIH Worldwide ISBN: p. 1-882417, 2002.

[15] Chan, A.P., et al., Determining an optimal recovery time after exercising to exhaustion in a controlled climatic environment: Application to construction works. Building and Environment, 56: p. 28-37, 2012.

[16] Liu, X. and Wang, X. Development of a smart work site for early warning of heat stress. in 20th International Symposium on Advancement of Construction Management and Real Estate. 2015. Hangzhou, China.

[17] Yi, W., et al., Development of an early-warning system for site work in hot and humid environments: A case study. Automation in Construction, 62: p. 101-113, 2016.

[18] Wagner, M.M., Moore, A.W., and Aryel, R.M., Handbook of biosurveillance. Academic Press, 2011.

[19] McQuiggan, S.W., Lee, S., and Lester, J.C., Predicting User Physiological Response for Interactive Environments: An Inductive Approach. AIIDE, 2006: p. 60-65, 2006.

[20] Besson, P., et al., Effectiveness of Physiological and Psychological Features to Estimate Helicopter Pilots' Workload: A Bayesian Network Approach. Intelligent Transportation Systems, IEEE Transactions on, 14(4): p. 1872-1881, 2013.

[21] Auffenberg, F., Stein, S., and Rogers, A., A personalised thermal comfort model using a Bayesian network. 2015.
[22] Bang-Jensen, J. and Gutin, G.Z., Digraphs: theory, algorithms and applications. Springer Science \& Business Media, 2008.

[23] Jensen, F.V., An introduction to Bayesian networks. Vol. 210. UCL press London, 1996.

[24] Jouffe, S.C.L., Bayesian Networks and BayesiaLab-A Practical Introduction for Researchers. USA. 383, 2015.

[25] Korb, K.B. and Nicholson, A.E., Bayesian artificial intelligence. CRC press, 2010.

[26] Liao, Y., et al., Risk assessment of human neural tube defects using a Bayesian belief network. Stochastic Environmental Research and Risk Assessment, 24(1): p. 93-100, 2010.

[27] ISO, I., Evaluation of Thermal Strain by Physiological Measurements. International Organisation for Standardisation, 1992.

[28] Moran, D., et al., Evaluation of the environmental stress index for physiological variables. Journal of thermal biology, 28(1): p. 43-49, 2003.

[29] Silver, A.J., et al., Effect of aging on body fat. Journal of the American Geriatrics Society, 41(3): p. 211-213, 1993.

[30] Matheron, G., Principles of geostatistics. Economic geology, 58(8): p. 1246-1266, 1963.

[31] Stytz, M.R. and Parrott, R.W., Using kriging for 3D medical imaging. Computerized Medical Imaging and Graphics, 17(6): p. 421-442, 1993.

[32] Oliver, M.A. and Webster, R., Kriging: a method of interpolation for geographical information systems. International Journal of Geographical Information System, 4(3): p. 313-332, 1990.

[33] Cressie, N., The origins of kriging. Mathematical geology, 22(3): p. 239-252, 1990. 